\title{
Visibility Trials of Unmanned Aerial Vehicles (Drones) by commercial $X$-Band Radar in Sub-urban Environment
}

\author{
Gaspare Galati ${ }^{(1)}$, Gabriele Pavan ${ }^{(1)}$, Francesco De Palo ${ }^{(1)}$, \\ Daniele Latini $^{(2)}$, Francesco Carbone ${ }^{(3)}$, Fabio Del Frate $^{(3)}$ Fabio Pietrobono $^{(4)}$ \\ ${ }^{1}$ Department of Electronic Engineering, “Tor Vergata” University, via del Politecnico 1 - Rome, Italy \\ gaspare.galati@uniroma2.it, gabriele.pavan@uniroma2.it, francesco.de.palo@uniroma2.it \\ ${ }^{2}$ GEO-K s.r.l., Via del Politecnico 1 - Rome, Italy; apr@geo-k.co \\ ${ }^{3}$ Department of Civil Engineering and Computer Science Engineering \\ "Tor Vergata" University, via del Politecnico 1 - Rome, Italy \\ fabio.del.frate@uniroma2.it; francesco.carbone@uniroma2.it \\ ${ }^{4}$ Free Lance Mechanical Engineer, fabio.pietrobono@gmail.com
}

\begin{abstract}
Preliminary experimental results obtained using a commercial $\mathrm{X}$-band radar to detect drones are described. Trials were carried out on April 1 1 st 2017 at the "Tor Vergata" University in Rome with the aim to compare the drone's echo to those coming from known reflectors in order to estimate the radar visibility of the drone.
\end{abstract}

Keywords-UAV, Drones, Radar Cross Section, Radar Trials, Corner Reflectors

\section{INTRODUCTION}

The term "drone" comes from the military world in which it is better addressed as Unmanned Aerial Vehicles (UAV). The UAV owes its success to its ability to carry a payload as well as to operate in very difficult environments, such as natural disasters, inaccessible regions and contaminated areas. Nowadays, thanks to cheaper cost as compared to the past, its use has been expanded to the civilian world with a number of applications only limited by the human's creativity. Journalists, filmmakers, farmers, scientists, hobbyists and many other use under-25kg drones in order to both reduce operational surveying costs and get innovative and attractive views of a particular area. The possibility of a drone to carry on a payload makes its use very interesting and at the same time potentially dangerous for people. In America the Federal Aviation Administration (FAA) regulations regarding drones, being used in any commercial application, are clearly defined while individuals flying for hobby or recreation are strongly encouraged to follow some safety guidelines [1]. In Italy, the guidelines related to the use of drones are released by National Agency for Civil Aviation (ENAC) [2]. In particular, the regulations distinguish between recreational use and commercial use. The latter imposes the UAV certification (after inspection by ENAC) and the pilot certification (for both theoretical and practical capabilities). Moreover, the flight volume is always limited to $500 \mathrm{~m}$ in range and $150 \mathrm{~m}$ in altitude from the pilot and only in the daily hours.
Concerning the recreational use, it is allowed only in authorized flight fields without any others requirements for pilots or vehicle. However, unfortunately, drones are not only attractive and useful for legal applications or recreation activities. In the very recent years the terrorism threat has grown making use of cheap and easy-to-find devices; in this framework a drone might be easily used to drop down small bombs or chemical substances on crowded environments where a big and expensive helicopter can't get access or, anyway, could be suddenly intercepted. An example could be found in [3], [4]. Then the problem of how the drones can be controlled over a limited area is strongly rising up. The first idea that comes in mind to face the problem is to reproduce the surveillance system already successfully used in the Air Traffic Control (ATC) by radar sensors. Air surveillance radars are very expensive and not easily deployable and also, operating at $\mathrm{S}$-band $(1-3 \mathrm{GHz})$, are suitable to detect big objects, like airplane, rather than small drones. On the other hand, drones might be seen by the cheaper commercial X-band $(9.2-9.5 \mathrm{GHz})$ marine radars operating with a wavelength of about $3 \mathrm{~cm}$. These radars have either a "solid state" transmitter or, more often, a magnetron transmitter with a transmitted peak power of tens of $k W$. Operating in Xband, they are more portable and deployable as compared with the big sized S-band air surveillance radars.

In this paper preliminary drone's visibility trails have been carried out by making use of an X-band marine radar in order to understand how a drone is seen by the radar (absolute measurement) and how it is compared with a well-known targets (relative measurement) when it flies at $400 \mathrm{~m}$ away from the radar. This preliminary trials don't address the drone's detectability - in the sense of radar detection - since for it a much deeper understanding of the particular radar chain must be carried out, beyond the goal of this paper. At the end of the paper only a simple consideration about the radar detection will be given. 
In the second section the radar will be detailed together with the method used to get the data from it (calibration). The third section will introduce the used targets, both corner reflectors and drone with given dimensions, and the sub-urban environment. Finally, in the fourth section the performed radar trials will be detailed and explained with the pertaining results.

\section{THE RADAR SENSOR AND ITS CALIBRATION}

The radar used for the trials is a commercial marine magnetron radar by the NAVICO company operating in X-band at $9.410 \mathrm{GHz}$. It is managed for research purposes by the Tor Vergata RadarLab group, under loan of use agreement with NAVICO. It transmits a peak power of $12 \mathrm{~kW}$ with different pulse lengths at different full-scales; we always use a full-scale of $0.25 N M$ (Nautical Miles), i.e. $463 \mathrm{~m}$, with a short pulse (50 $\mathrm{ns}$ ) providing a $7.5 \mathrm{~m}$ range resolution. In Fig. 1 the simplified block diagram of the radar's receiver is shown.

\section{Simplified block diagram of the radar receiver}

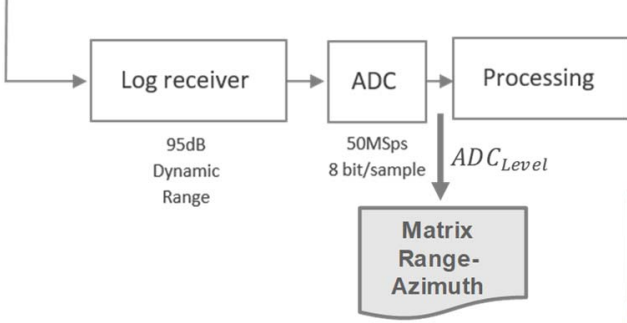

$[\mathrm{R}, \theta]$

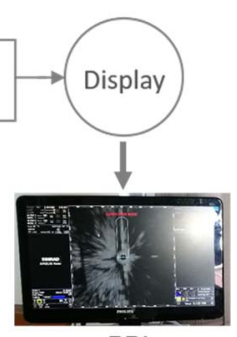

PPI
Fig. 1. Simplyfied block diagram of the radar's receiver.

The antenna is a 6-feet horizontally polarized slotted waveguide antenna rotating at 24 RPM (Revolutions Per Minute) with a maximum gain of $29 d B$. The logarithmic receiver compresses the $95 d B$ input dynamic into a voltage range of $0-2 \mathrm{~V}$, then converted by the 8-bit Analog-to-DigitalConverter (ADC). Just after the ADC it is possible to catch the Range-Azimuth matrix $[R, \theta]$ containing all the acquired levels. Before being sent to Plane-Position-Indicator (PPI) display, the video signal is adjusted by the gain stage that normalizes the image histogram and sets the minimum level to be displayed: the greater the gain, the lower the minimum displayed level. Table I shows the main radar parameters.

TABLE I. RADAR PARAMETERS

\begin{tabular}{|c|c|c|}
\hline Parameters & Value & Value [dB] \\
\hline Transmitted power $P_{t x}$ & $12 \mathrm{~kW}$ & $40.8 \mathrm{dBW}$ \\
\hline Gain: $G_{t x}, G_{r x}$ & \multicolumn{2}{|c|}{$29 \mathrm{~dB}$} \\
\hline Wavelength $\lambda$ & $3.19 \mathrm{~cm}$ & $-14.96 \mathrm{~dB}$ \\
\hline Loss $L^{(*)}$ & \multicolumn{3}{|c|}{$5 \mathrm{~dB}^{(*)}$} \\
\hline
\end{tabular}

${ }^{*}$ It is necessary to distinguish between received power loss (due to only analogic components up to logarithmic amplifier) and detection loss, the latter includes processing loss (i.e. sampling, ADC, CFAR, IR if active, binary integrator, ... etc.).
In Fig. 2 the photos of the radar set (left) and the radar console with PPI video (right) are shown.
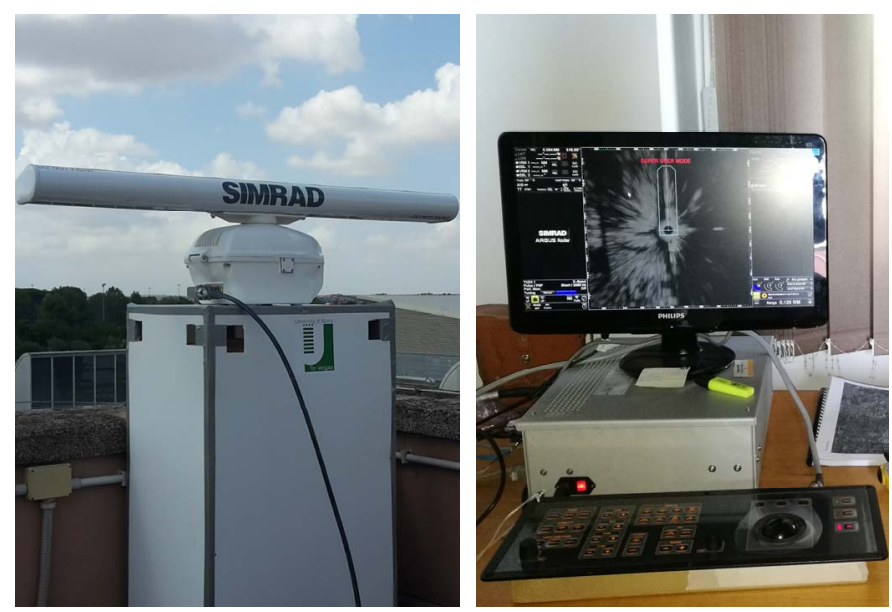

Fig. 2. Radar body on moving platform (left) and console with PPI (right).

The radar is developed for marine environment in which, for a given target, the movement of pitch of the vessel should not modify the received power [5]. Hence, the antenna pattern is broad in elevation with a $-3 d B$ beam-width of $\pm 12^{\circ}$. The horizontal beam-width is $1.35^{\circ}$ so that at $400 \mathrm{~m}$ the angular resolution is $9.5 \mathrm{~m}$. Since the $[R, \theta]$ matrix is recordable before any signal processing, its levels are fully representative of the received power through the following relationship:

$$
P_{r x}[d B m]=\alpha \cdot\left(A D C_{\text {Level }}-L_{\text {noise }}\right)-P_{\text {noise }}[d B m]
$$

where $A D C_{\text {Level }}$ is the level read from the recorded matrix for the particular range cell, $L_{\text {noise }}$ is the noise floor level corresponding to the power $P_{\text {noise }}$ (in $\mathrm{dBm}$ ), the latter depends on the selected pulse mode (short, medium, long), while $\alpha(d B /$ Level $)$ is the step associated to each level.

To calibrate the radar, i.e. to evaluate $\alpha$ and $L_{\text {noise }}$, we use the level and the power associated to the noise and to the saturation. We acquired only samples of noise transmitting short pulses of $50 \mathrm{~ns}$, i.e. with a band $B=20 \mathrm{MHz}$. The mean noise power is $P_{n}=F k_{B} T B$. Supposing a noise figure $F=$ $5 \mathrm{~dB}$, a system temperature $T=290 \mathrm{~K}$, with $k_{B}=1.38 \times$ $10^{-23} \mathrm{~J} / K$ the Boltzmann's constant: $P_{n}=-96 \mathrm{dBm}$. On 21000 ADC samples of noise, the estimated mean level resulted 23. Subsequently we acquired an entire PPI of samples (1687950) due to echoes of noise, clutter, near or far buildings, towers, lampposts, etc.. We expected that the presence of near large buildings (less than $300 \mathrm{~m}$ from radar) sends in saturation the logarithmic amplifier. Observing the histogram of the levels we have estimated 192 as the saturation level, which corresponds a saturation power of $-15 \mathrm{dBm}$ given by the manufacturer. So on the plane (Level, $P_{r x}$ ) two points $A \equiv$ $(23,-96)$ and $B \equiv(192,-15)$ are defined. Considering the straight line passing for $A$ and $B$, it results $\alpha=0.47929$ with $L_{\text {noise }}=23$ and Eq. (1) becomes:

$$
P_{r x}[\mathrm{dBm}]=0.47929 \cdot A D C_{\text {Level }}-107
$$

with $P_{\text {noise }}=-96 \mathrm{dBm}$. 


\section{TARGETS AND ENVIRONMENT}

In this section the available targets and the sub-urban environment are introduced.

\section{A. Corner Reflectors}

Three octahedral Corner Reflectors (briefly CR) were built for the trials (see Fig. 3). The large size CR weights $3.8 \mathrm{~kg}$, the medium one weights $900 \mathrm{~g}$ while the small CR weights $200 \mathrm{~g}$. The dimensions (side $a$ ) are scaled of a factor 2 from large to medium and from medium to small.

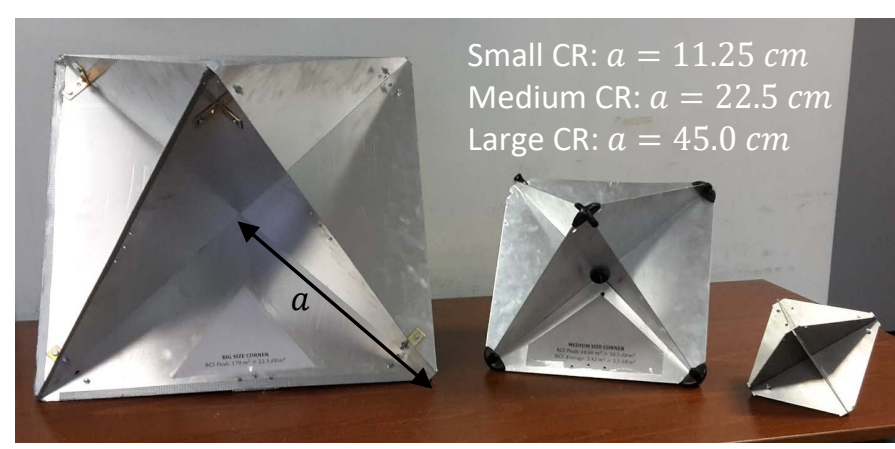

Fig. 3. Octhaedral corner reflectros: large, medium and small size.

As comparison, we consider a single trihedral (see Fig. 4). The theoretical peak of the Radar Cross Section (RCS) is $\frac{4 \pi a^{4}}{3 \lambda^{2}}$ (viewing perspective) when $\theta=\frac{\pi}{4}$ and $\psi=\tan ^{-1}\left(\frac{1}{\sqrt{2}}\right) \cong$ 35.26 deg [6], [7], [8].

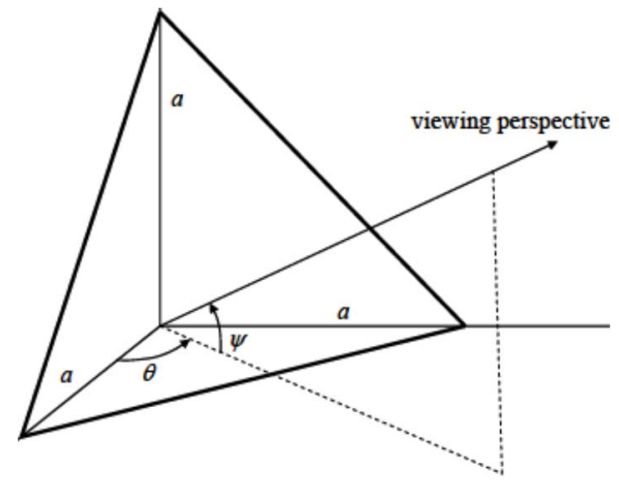

Fig. 4. Trihedral Reflector.

Considering the dimensions $a$ of the three CRs, the RCS peak of an equivalent trihedral are for small, medium and large: $0.659 \mathrm{~m}^{2}\left(-1.8 \mathrm{dBm}^{2}\right), 10.56 \mathrm{~m}^{2}\left(10.2 \mathrm{dBm}^{2}\right)$ and $169 \mathrm{~m}^{2}$ $\left(22.2 \mathrm{dBm}^{2}\right)$ respectively. Normally in vessels, for its simple installation, the CR is mounted in the "upright" position where the axis of symmetry is vertical (see Fig. 5a). Starting from "upright" position with azimuthal angle $\theta_{0}=45^{\circ}$, the CR mounted in "catch rain" (see Fig. 10b) can be obtained rotating (in depression) of an elevation angle $\psi_{0}=\operatorname{arctg}(\sqrt{2}) \cong$ $54.74^{\circ}$. In this way, observing the $\mathrm{CR}$ along a horizontal plane passing for the CR centre, the RCS can be approximated by the one corresponding to a trihedral observed with an elevation of $\psi_{0}=54.74^{\circ}$. It results $\operatorname{RCS}\left(\theta_{0}, \psi_{0}\right)=\frac{R C S_{\text {peak }}}{2}$.
It is preferable to use the CR mounted in "catch rain" being the RCS less sensitive to the elevation angle $\psi$.

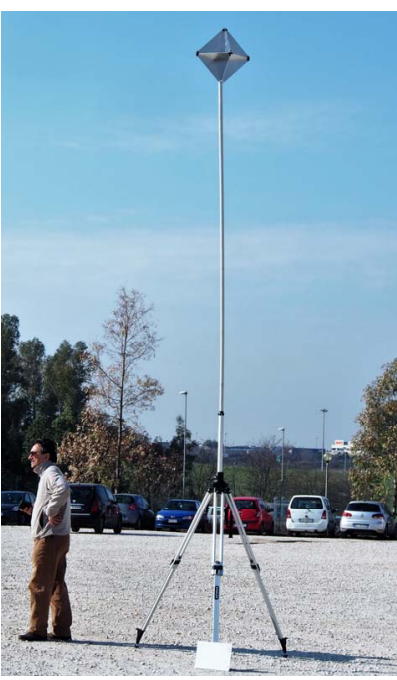

(a)

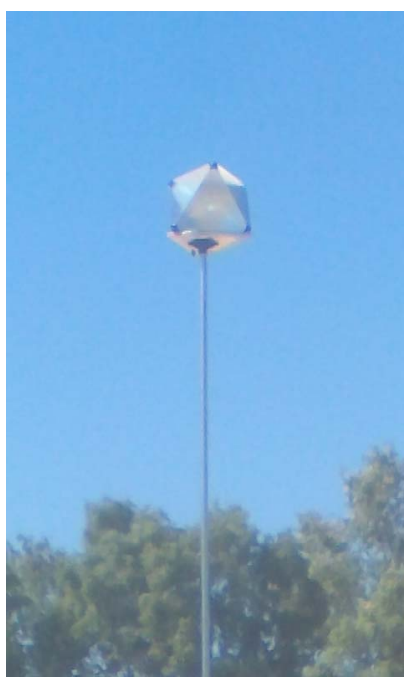

Fig. 5. Medium size CR on a $5.4 \mathrm{~m}$ pole facing the radar: (a) "upright" mounted, (b) "cacth rain" mounted.

Table II shows the RCS of CRs in "catch rain" position, approximated using a trihedral reflector.

TABLE II.

RCS OF CRS IN “CATCH RAIN” POSITION USING TRIHEDRAL APPROXIMATION

\begin{tabular}{|c|c|c|c|}
\hline CR Type & $a[m]$ & $\mathrm{RCS}_{\text {peak }}\left[\mathrm{m}^{2}\right]$ & $\mathrm{RCS}_{\text {peak }}\left[\mathrm{dBm}^{2}\right]$ \\
\hline Small & 0.1125 & 0.3295 & -4.8 \\
\hline Medium & 0.225 & 5.28 & 7.2 \\
\hline Large & 0.450 & 84.5 & 19.2 \\
\hline
\end{tabular}

\section{B. Drone}

The UAV exploited for this test was an exa-rotor with brushless engines, lightweight with high quality carbon fiber frame, as shown in Fig. 6. The drone's maximum diameter is $115 \mathrm{~cm}$ with open propellers and it is $42 \mathrm{~cm}$ high. Each brushless rotor had two propellers, each $15 \mathrm{~cm}$ long. The flight can be accomplished in manual, GPS or automatic mode.

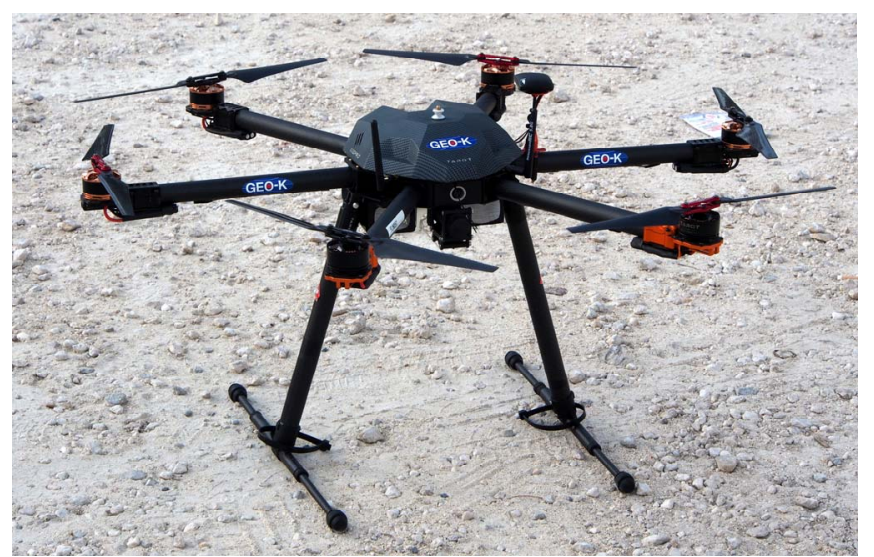

Fig. 6. Drone used for the trials. 
Considering the latter case, the flight plan is properly defined during the briefing phase, through a specific software installed in the ground station. Once in the air, the UAV carries the preset mission and strictly follows the flight plan until landing, again automatically, at the established point.

The vehicle has $5.6 \mathrm{~kg}$ net weight with $4 \mathrm{~kg}$ of maximum payload. It ensures 20 minutes of endurance flying up to $18 \mathrm{~km} / \mathrm{h}$ with the most innovative fail-safe technologies and the high degree of stability also on severe wind conditions (up to $30 \mathrm{~km} / \mathrm{h}$ wind gust). The maximum operational range is $2 \mathrm{~km}$ under radio control with an altitude limit of $150 \mathrm{~m}$ as imposed by the authorities. Moreover, as strictly required in Italy, it has been certified by ENAC for operations in critical scenarios.

\section{Sub-urban environment}

Trials have been performed on day April $1^{\text {st }} 2017$ at "Tor Vergata" University in Rome (Italy). The radar was deployed on the roof of the Information Engineering building while the targets were placed in the parking lot between Literature and Economics Faculties, as shown in Fig. 7.

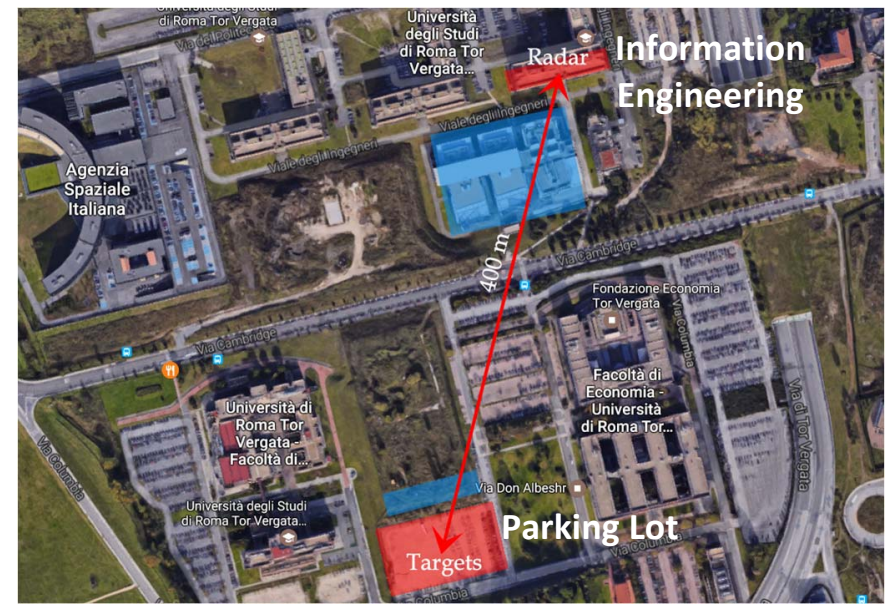

Fig. 7. Map of the surrounding environment: targets at $405 \mathrm{~m}$ from the radar's location.

The choice of the parking lot as targets' location was driven both by the line-of-sight propagation (see Fig. 8) and by the absence of heavy clutter in that place as shown in Fig. 9 where the PPI map of the acquired environment is shown. It is possible to recognize the parking lot as the place among the three highlighted lampposts.

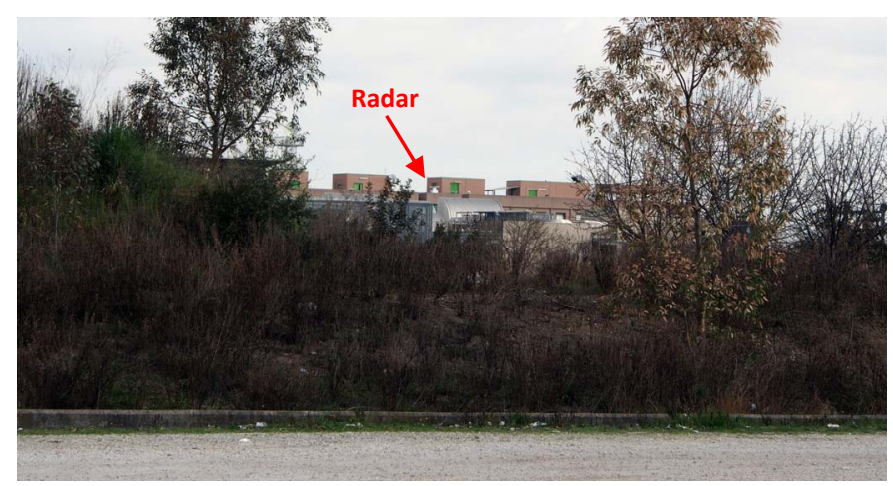

Fig. 8. Line-of-sight propagation between the radar and the parking lot.
In order to get rid of parked cars the parking lot was closed during the trials. The surface clutter at the place of the parking lot was $-79.4 \mathrm{dBm}$, that is the level of the black color among the three lampposts in Fig. 9. It is to be compared with a receiver noise level of $-96 \mathrm{dBm}$.

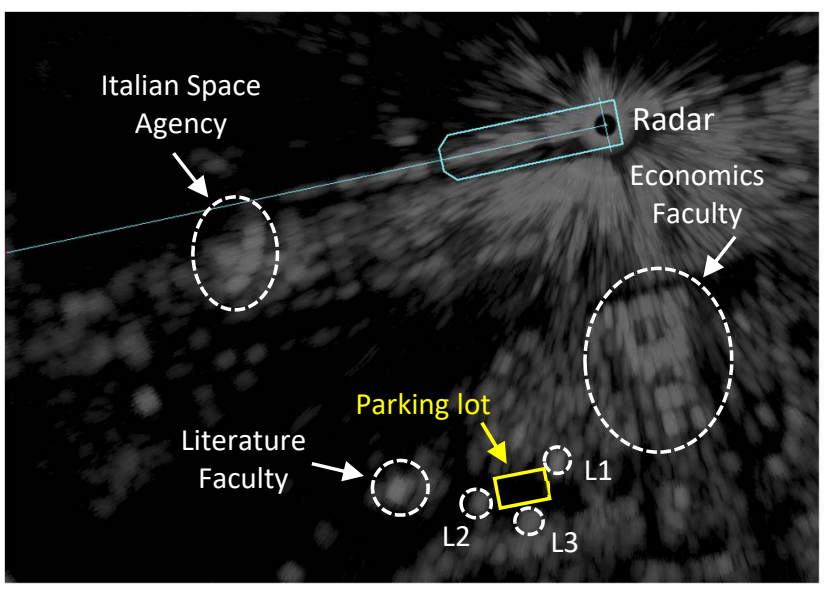

Fig. 9. PPI of the environment: the parking lot is placed among the lampposts.

\section{TRIALS}

The main goal of the trials was to compare the drone's echo to those coming from known reflectors in order to estimate its RCS. The received power and the RCS $\sigma$ are theoretically related by the radar equation [9], [10]:

$$
P_{r x}=\frac{P_{t x} G_{t x} G_{r x} \lambda^{2} \sigma}{(4 \pi)^{3} R^{4} L}
$$

for which the useful parameters are shown in Table I.

Firstly one at the time, the three CRs were placed in the middle of the parking lot raised up to a $5.4 \mathrm{~m}$ pole, as shown in Fig. $5 \mathrm{~b}$ for medium CR. The CR was oriented in azimuth in order to provide the maximum RCS. The recorded radar PPI image is shown in Fig. 10.

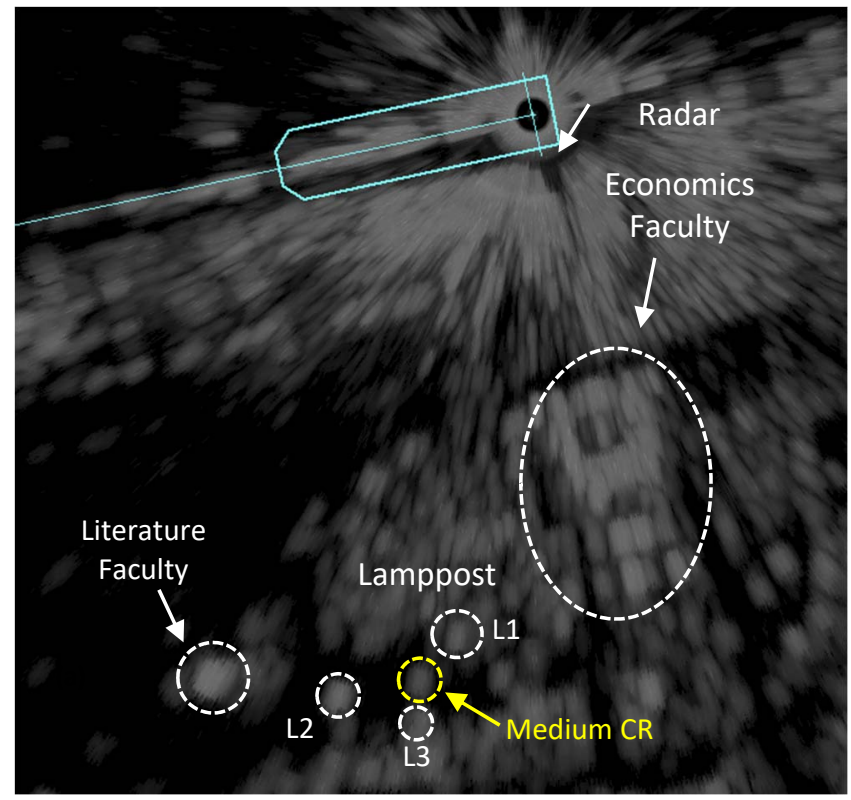

Fig. 10. PPI of medium size $\mathrm{CR}$ at $h=5.4 \mathrm{~m}: P_{r x}=-47.8 \mathrm{dBm}$. 
Inverting Eq. (3), for small, medium and large CR the power and the RCS result respectively: $\left(-49.1 \mathrm{dBm},-5.7 \mathrm{dBm}^{2}\right)$, $\left(-36.6 \mathrm{dBm}, 6.8 \mathrm{dBm}^{2}\right)$ and $\left(-25.1 \mathrm{dBm}, 18.4 \mathrm{dBm}^{2}\right)$. Fig. 10 shows the estimated RCS of the CR in comparison with the RCS of the trihedral approximation (see Table II).

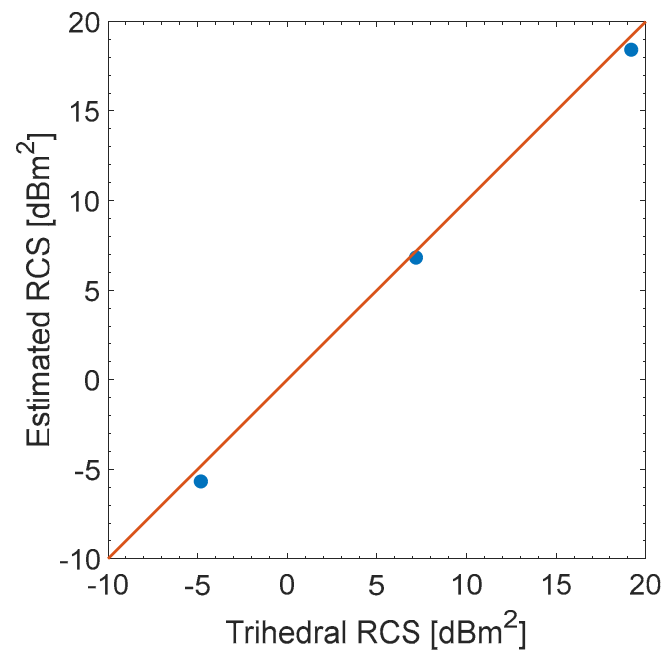

Fig. 11. Estimated RCS of the three CRs vs the Trihedral approximation.

Being the radar equation in Eq. (3) not fully aware of the real environment neither of the particular radar's internal behavior (e.g. losses, supposed here of $5 d B$ ), we don't expect to perfectly match the practical results with the theoretical expectation, however they are very close to the theoretical ones. As expected, the difference between the received power (or RCS) levels of the three CRs (large-medium and medium-small) is around $12 d B$, very close to the theoretical one of $12 d B$ due to $1: 2$ size ratio.

After the CRs' acquisitions, the drone (without payload) flew at the same height of $5.4 \mathrm{~m}$ above ground level. The acquired PPI image is shown in Fig. 12.

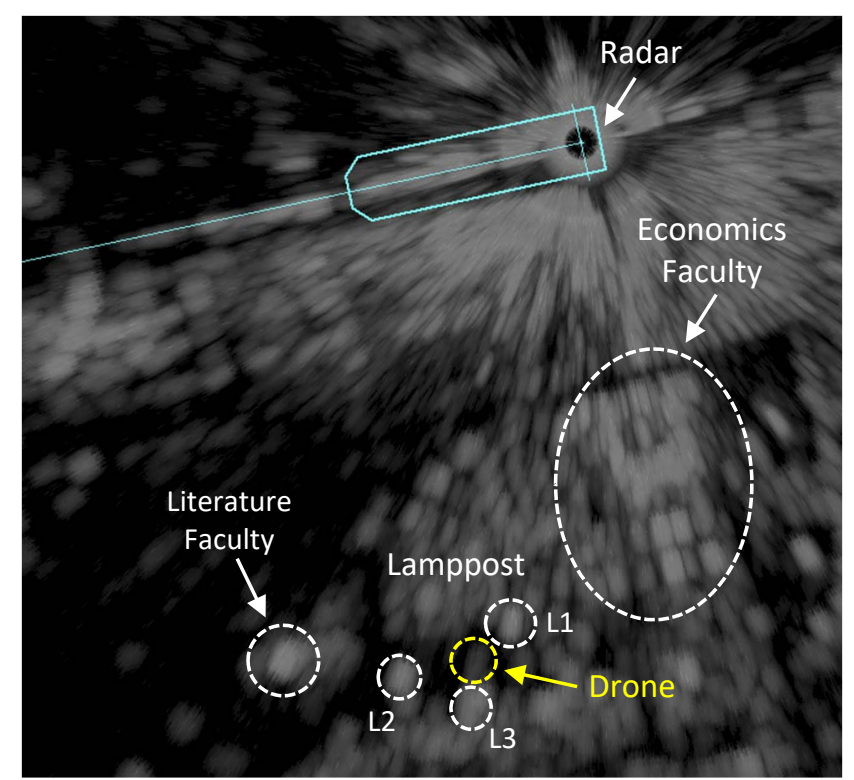

Fig. 12. PPI of drone at $h=5.4 \mathrm{~m}: P_{r x}=-46.6 \mathrm{dBm}, R C S=-3.1 \mathrm{dBm}^{2}$.
The received power was $-46.6 \mathrm{dBm}, 2.5 \mathrm{dBm}$ greater than the small CR. In order to make the drone more visible by the radar, the small size CR was deployed under its body, as shown in Fig. 13. The small CR is very light in order to don't load too much the drone.

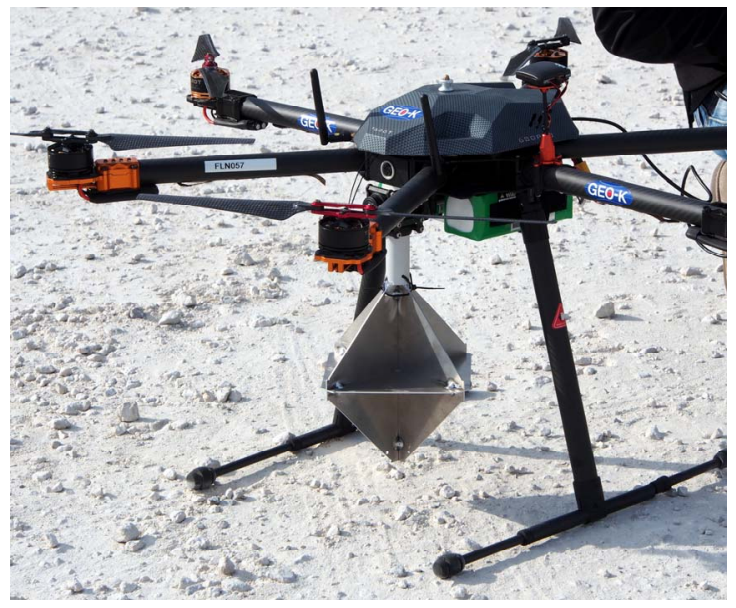

Fig. 13. Small size CR mounted under the drone's body.

The drone flew at the height of $5.4 \mathrm{~m}$ in order to be comparable with the previously performed measurements. Due to the added small CR, we expected to get more power from this target as compared with that of the drone alone. From Table III the improvement of the drone RCS with incorporated small CR is around $3.9 d B$.

In a successive trial the drone flew at the height of $40 \mathrm{~m}$ with and without payload (i.e. the small CR). The results are shown in Table III. The RCS considerably decreases. However, we observe that the small oscillations of the drone, caused by the weak wind that it tried to balance, did not allow a stable position of the small CR. Because of this drawback we had no control over the CR's RCS which fluctuated, making very difficult a deterministic measurement: drones are fluctuating targets.

TABLE III. RECEIVED POWER LEVELS AND RCS

\begin{tabular}{|l|c|c|}
\hline \multicolumn{1}{|c}{ Target } & $\begin{array}{c}\text { Measured } \\
\text { Power }(\mathrm{dBm})\end{array}$ & $\begin{array}{c}\text { Estimated } \\
\text { RCS }\left(\mathrm{dBm}^{2}\right)\end{array}$ \\
\hline Noise level & -96.0 & - \\
\hline Clutter & -79.4 & - \\
\hline Small CR @ 5.4m & -49.1 & -5.7 \\
\hline Medium CR @ 5.4m & -36.6 & 6.8 \\
\hline Large CR @ 5.4m & -25.1 & 18.4 \\
\hline Drone @ 5.4m & -46.6 & -3.1 \\
\hline Drone @ 40m & -55.2 & -11.7 \\
\hline Drone with Small CR @ 5.4m & -42.7 & 0.7 \\
\hline Drone with Small CR @ 40m & -52.0 & -8.5 \\
\hline
\end{tabular}

In conclusion, from the measurements in X-band, it results an estimation of the drone's RCS from $-11.7 \mathrm{dBm}^{2}$ to $-3.1 \mathrm{dBm}^{2}$. As comparison a swan provides, in X-band, a RCS of $-16 \mathrm{dBm}^{2}$ [11], e.g. $0.025 \mathrm{~m}^{2}$. 
Table IV reports some RCS of targets in the sub-urban environment around the Tor Vergata area (see also Fig. 14). The last column of Table IV shows, as comparison, the mean RCS of some ships. Fig. 14 shows an overlay between the Google Earth map with the RCS map.

TABLE IV.

RCS OF TARGETS IN THE SUB-URBAN ENVIRONMENT AND COMPARISON WITH RCS OF SHIPS

\begin{tabular}{|l|c|c|c|c|c|}
\hline \multicolumn{1}{|c|}{ Target } & $\begin{array}{c}\text { Range } \\
(\mathbf{m})\end{array}$ & $\begin{array}{c}\text { Prx } \\
(\mathbf{d B m})\end{array}$ & $\begin{array}{c}\text { RCS } \\
\left(\mathbf{m}^{2}\right)\end{array}$ & $\begin{array}{c}\text { RCS } \\
\left.(\mathbf{d B m})^{2}\right)\end{array}$ & \multicolumn{1}{|c|}{ Ships } \\
\hline $\begin{array}{l}\text { Street } \\
\text { Lamp L1 }\end{array}$ & 432 & -32.2 & 17.3 & 12.4 & $\begin{array}{c}\text { Cabin } \\
\text { Cruiser }\end{array}$ \\
\hline $\begin{array}{l}\text { Street } \\
\text { Lamp L2 }\end{array}$ & 369 & -22.6 & 83.7 & 19.2 & Coaster \\
\hline $\begin{array}{l}\text { Building } \\
\text { Literature }\end{array}$ & 468 & -18.7 & 524 & 27.2 & $\begin{array}{l}\text { Coast } \\
\text { Guard }\end{array}$ \\
\hline $\begin{array}{l}\text { General } \\
\text { Hospital }\end{array}$ & 540 & -19.7 & 745 & 28.7 & Freighter \\
\hline $\begin{array}{l}\text { Building } \\
\text { B1 }\end{array}$ & 396 & -17.3 & 374 & 25.7 & $\begin{array}{l}\text { Small } \\
\text { Cargo }\end{array}$ \\
\hline $\begin{array}{l}\text { Building } \\
\text { B2 }\end{array}$ & 1296 & -33.6 & 1004 & 30.0 & Tanker \\
\hline $\begin{array}{l}\text { Rectorate } \\
\text { Building }\end{array}$ & 2268 & -27.8 & 35454 & 45.5 & Container \\
\hline
\end{tabular}

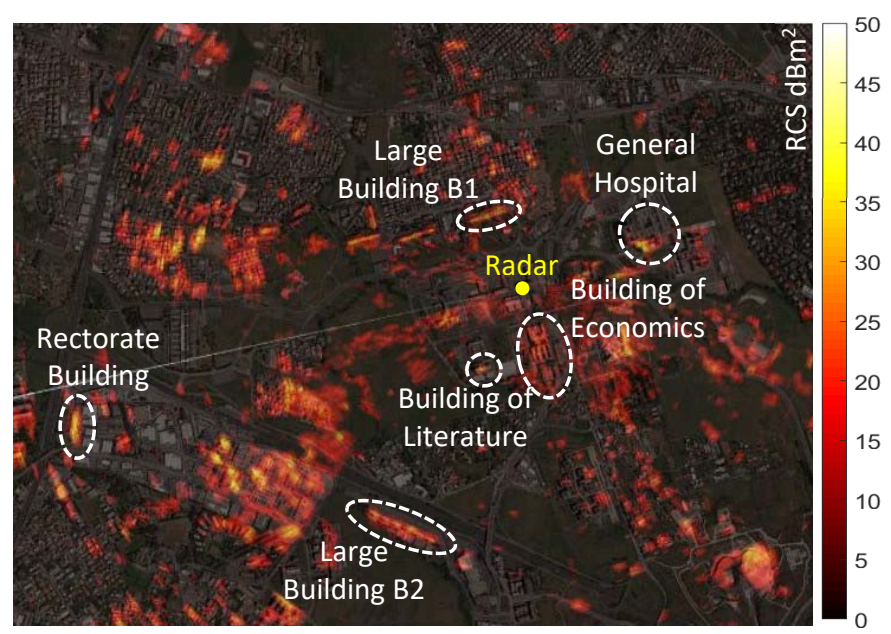

Fig. 14. RCS map of Tor Vergata area.

\section{CONCLUSION}

In this paper preliminary radar trials with a drone have been described. The trials took place in April 2017 at "Tor Vergata" University in Rome (Italy). The major aim was to get a rough estimation of the Radar Cross Section of a drone in an area with little clutter inside a sub-urban environment. Trials did not take into account the radar detection process for which further measurements campaign are going to be scheduled. The radar was a commercial marine magnetron radar in X-band. The trials were carried out in a comparative way between the drone and well-known corner reflectors. The well-known radar targets used as reference were three (small, medium and large sized) octahedral corner reflectors of different RCS while the drone was equipped so that the small CR could be mounted under its body. As first test, the power received from the three CRs were recorded in order to perform the calibration of radar. As expected the difference in received power for large-medium and for medium-small was of about $12 \mathrm{~dB}$, exactly the same amount in RCS difference. After that, the drone was let to fly at the same height of CRs $(5.4 \mathrm{~m})$ in order to compare its return with those coming from the CRs and, subsequently at the height of $40 \mathrm{~m}$. The result has given an estimation of the drone's RCS in X-band into a range from $-11.7 \mathrm{dBm}^{2}$ to $-3.1 \mathrm{dBm}^{2}$.

The trials described in this paper are a preliminary task which will be followed by other trials in which the radar detection process might be considered for the drone. The key point is the ability of a not-expensive and compact commercial marine radar to detect, and give alarm for, an unauthorized flying drone.

\section{ACKNOWLEDGMENT}

The authors are grateful to Mr. Sergio Pandiscia for having taking care of the logistic operations that made possible the trials, to NAVICO which provided the radar and to the FlyTop company from which the drone has been acquired. Also we would like to thank Dr. Emiliano Coha and Ten. Andrea Proietti of the Security Service of the University who closed the parking lot to the traffic.

\section{REFERENCES}

[1] Unmanned Aircraft Systems: https://www.faa.gov/uas [Online].

[2] Remotely Piloted Aerial Vehicles Regulation - Issue N. 2, 16 July 2015, Revision 2, December 2016. https://www.enac.gov.it [Online].

[3] Drone attack in Pakistan: 2005-2017.

www.satp.org/satporgtp/countries/pakistan/database/Droneattack .htm," [Online].

[4] Drone attack in Pakistan: http://www.pakistanbodycount.org [Online].

[5] J. N. Briggs, Target Detection by Marine Radar, London: The Institution of Electrical Engineers, 2004.

[6] A. W. Doerry, B. C. Brock, Radar Cross Section of Triangular Trihedral Reflector with Extended Bottom Plate, SANDIA REPORT SAND2009-2993, May 2009.

[7] S. Luke, Performance investigation of marine radar reflectors on the market, QINETIQ/D\&TS/SEA/CR0704527/2.0. March 2007.

[8] E. F. Knott, J. F. Shaeffer and M. T. Tuley, Radar Cross Section, Scitech Publishing, 2004.

[9] D. K. Barton, Radar Equations for Modern Radar, Artech House, 2013.

[10] M. Skolnik, Radar Handbook, McGraw Hill, 2008.

[11] C. R. Vaughn, Birds and insects as radar targets: A review. Proceedings of the IEEE, Vol. 73, N. 2, February 1985. 